CLAWAR 2018: 21st International Conference on Climbing and Walking Robots and the Support Technologies for Mobile Machines, Panama City, Panama, 10-12 September 2018

\title{
STANDING ASSISTANCE THAT CONSIDERS USER POSTURE TOLERANCE
}

\author{
M.YOKOTA*, S. KAWAZOE*, D. CHUGO* \\ * Graduate School of Science and Technology, Kwansei Gakuin University, \\ Sanda, Hyogo, Japan \\ E-mail: \{yokota, kawazoe, chugo\}@chugolab.com \\ www.chugolab.com \\ S. YOKOTA** and H. HASHIMOTO*** \\ ** Toyo University \\ Kawagoe, Saitama, Japan \\ *** Advanced Institute of Industrial Technology \\ Shinagawa, Tokyo, Japan
}

\begin{abstract}
This paper proposes a novel standing assistance robot, which considers the posture tolerance of its user. In previous studies, conventional assistive robots did not require patients to use their own physical strength to stand, which leads to decreased strength in the elderly. Therefore, an assistive robot that allows patients to maximally use the physical strength they possess is required. To realize this objective, it is important that a robot assists patients according to their body movement and by their intentions. However, in previous studies, general assistive robots helped patients by using a fixed motion reference pathway in spite of their original intention, and as a result, these robots failed to use the physical strength of the patients. Therefore, we propose a novel standing assistance robot, which allows its user to move their body within a prescribed degree of posture tolerance during the process of moving from a sitting to a standing position. Our key findings cover two fundamental research topics. One is the investigation into posture tolerance during a standing motion. The other topic is a novel assistance control algorithm that considers the investigated posture tolerance by combining position control and force control. A prototype assistive robot, based on the proposed idea was fabricated to help patients stand up safely using the maximum of their remaining physical strength.
\end{abstract}

Keywords: Standing Assistance; Posture Tolerance; Robotic Walker.

\section{Introduction}

Standing is one of the most serious and important activities in the day-today lives of elderly people, as they may lack physical strength and stability $^{1} .^{2}$ In a typically bad case, an elderly person who does not have enough 
physical strength will not be able to stand up and may then be restricted to life in a wheelchair life or become bedridden. ${ }^{3}$ Furthermore, once an elderly person falls into such lifestyle, the decrease in their physical strength becomes more pronounced due to the lack of exercise and the associated limited use of their own physical strength. ${ }^{4}$ Therefore, assistive robots are required to aid patients in the use of their remaining strength during the standing motion in order to maintain their muscular strength.

To realize this objective, assistive robots should be designed to accept some variance in the patient's body motion because human motion does not always fit an established reference path. Despite this, there have been many reports of robots, which assist patients according to a fixed reference path and do not consider the variations in body motion. ${ }^{56}$ During standing, these robots interfered with the body motion of the patient, adjusting it to fit the reference path. This external intervention prevents patients from using their own physical strength during the process of standing up and therefore reduces their muscular strength.

Previously, we have developed robotic walkers which have a standing assistance function, ${ }^{789}$ and Fig. 1 shows our recent latest prototype. ${ }^{9}$ The robotic walker consists of a powered walker and a standing support manipulator with armrests, which moves the patient in an upward direction so as to be lifted. Wheel actuators on the powered walker stabilize the patient, and are also used for lifting the patient up. Our prototype succeeded to assist the patient in a way that enabled them to use their remaining physical strength as seen in Fig.1(b). However, this prototype did not consider the range in movement of the patient's body during standing up, and as a result, if the patient's motion does not fit the designed standing motion pathway, the prototype cannot make use of the remaining physical strength of the patient, as seen in Fig.1(c).

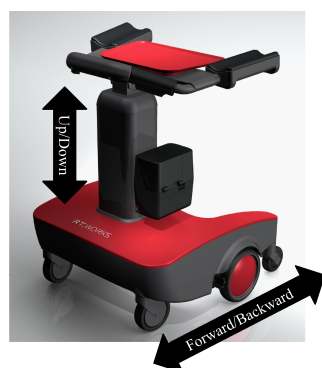

(a) prototype

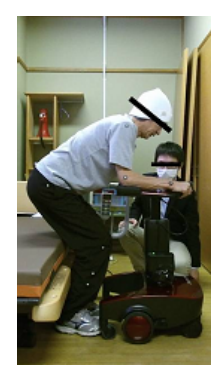

(b) success to assist

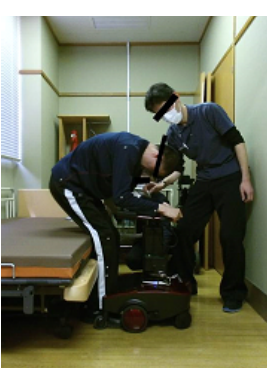

(c) failure to assist

Fig. 1. Our prototype which assists a patient who requires daily care. 
Therefore, in this study, we propose a novel standing assistance robot, which considers the variation in the range of movements of a patient's body when rising from a sitting to a standing position. To achieve this objective, we initially investigated the posture tolerance during the process of standing up. In this range, patients can stand up, stably and safely, using their own physical strength. Secondly, we extend a published assistance algorithm, ${ }^{8}$ which combines position control and force control, to adapt to the parameters of the prior investigation into posture tolerance. Using the proposed algorithm, our robotic device only assists them to stand up when necessary; maximizing their muscle exercise.

\section{Posture Tolerance}

\subsection{Required Condition}

From the viewpoint of body dynamics, the standing posture should be fulfilled by two conditions.

- Stability condition: The patient should be able to keep their body balanced in this posture. This study defines the condition as follows: the position of the center of gravity (COG) should be located within the range of the patients footprint, while keeping the body balanced during standing up. ${ }^{7}$

- Muscle condition: The patient should be able to control their body motion in this posture. In general, the output force generated by muscles, changes according to the human posture because the positional relationship between the muscles and bones changes with the adopted posture. ${ }^{8}$ This means an unsuitable posture cannot generate a sufficient upper direction output force for proceeding through with the standing motion. This study defines this condition as follows: the output force of the muscles listed in Fig.2 should not exceed the muscle's maximum output during standing.

This paper investigates the tolerance level, which fulfills these two conditions through computer simulation studies using OpenSim, a human motion dynamics simulator package. In this simulation, we used a 3DGaitModel $2392^{10}$ as human model and modified its body parameters to fit a typical Japanese elderly person. ${ }^{11}$ The standing motion was based on the references recommended by nursing specialists ${ }^{8}$ as shown in Fig.3(b). In Fig.3(b), the Y-axis shows the angular values of the pelvis and trunk, knee and ankle, whereas the $\mathrm{X}$-axis shows the movement pattern, ${ }^{7}$ which is the ratio of the standing motion, as shown by (1). 


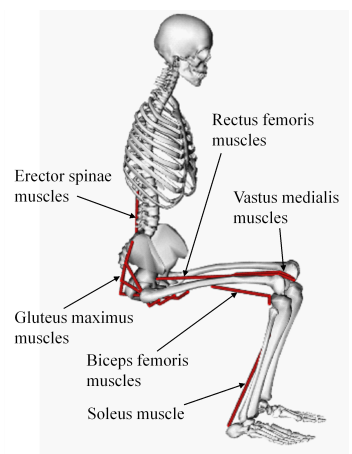

(a) muscles

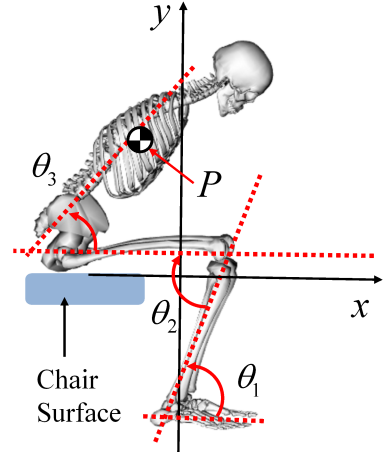

(b) coordination

Fig. 2. Human model

$$
\hat{s}=t / t_{s}
$$

In equation $1(1), t_{s}$ is the time required for completion of the standing-up operation and $t$ is the present time. Generally, the standing motion consists of three phases, as shown in Fig.3(a). Thus, we set a variation of $\pm 30[\mathrm{deg}]$ range on the reference posture at the end of each phase (Postures (A)(C)) in the computer simulation. Note posture (D) is the final posture and therefore we did not set a variation on this phase.

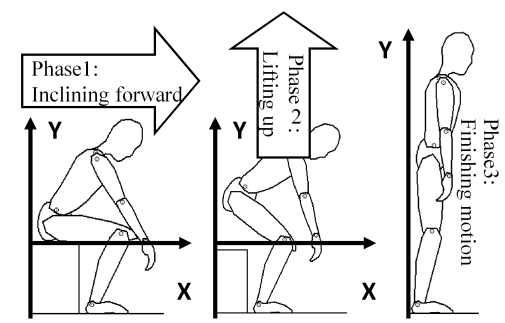

(a) standing phases

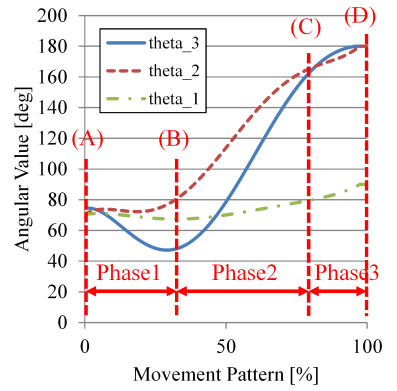

(b) references

Fig. 3. Simulation setup 


\subsection{Body Motion Tolerance}

Fig. 4 shows the acceptable position of point P, identified in Fig.2(b), derived from the computer simulation. The standing motion will be realized within the tolerance shown in Fig.4(a) and in this range of motion, the patient can physically achieve final standing posture.

Fig.4(b) shows the acceptable tolerance at 70[\%] movement during the pattern of the standing motion. Muscle condition determines the stability condition in Fig.4(b), and from this result, it is important to consider the patient's capable muscle output force in the standing posture.

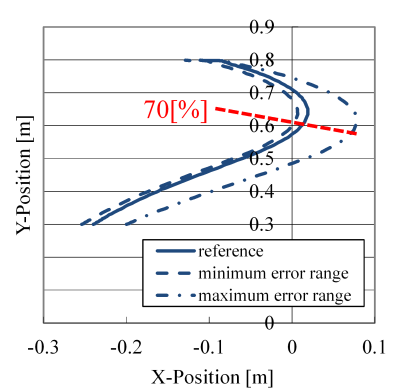

(a) Investigated tolerance

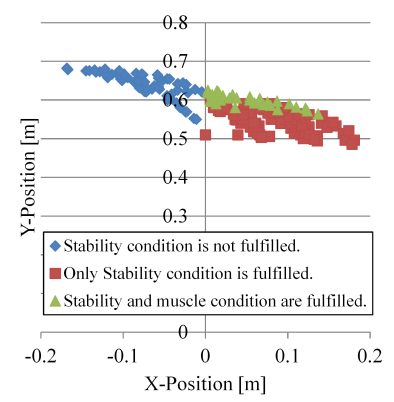

(b) Tolerance at $70[\%]$ movement pattern

Fig. 4. Simulation Results

\section{Assistance Control Algorithm}

To allow patients to use their remaining physical strength during standing up, our controller uses a combination of damping control and position control. Damping control can change the strength of assistive power, thus, it can determine how strong it applies assistance force to reduce load or use their remaining physical strength. Furthermore it allows for an offset from the reference pathway of motion, allowing the patient to move freely during the standing up process. Considering these characteristics, damping control should be used in the tolerance discussed in section two. By contrast, position control is useful for maintaining body posture, however, its pathway is fixed and so does not consider the physical strength of the patient. Thus, it is useful when the patient's posture exceeds the acceptable range.

In our previous work, ${ }^{8}$ we proposed an assistance control algorithm based on the voluntary movement of the patient. We know from previous research $^{12}$ that the motion of the human body consists of voluntary movements, which generate the total body motion, and a posture adjustment 
action, which keeps the body stable during motion. This means the robot should only provide a force that assists the physical activity in response to the voluntary movement of the patient, and our proposed algorithm only assisted the patient when physical strength was required for doing a voluntary movement. However, the previously reported algorithm did not consider the variation in the range of movements during human motion, so this paper extends this control algorithm as follows:

- First, we defined the body movement vector $\mathbf{P}$ as (2). This shows the velocity direction of point $\mathrm{P}$ (Fig.2(b)), which is the COG of the upper body. The position of $\mathrm{P}\left(x_{p}^{r e f}, y_{p}^{r e f}\right)$ is a motion reference point based on the standing motion recommended by nursing specialists. Details regarding the generation of this reference point are given in our previous paper. ${ }^{8}$

$$
\begin{aligned}
& \mathbf{P}=\mathbf{v}_{p}^{r e f}(\hat{s}), \\
& \mathbf{v}_{p}^{r e f}=\left|\begin{array}{l}
\dot{\mathbf{x}}_{p}^{\text {ref }} \\
\dot{\mathbf{y}}_{p}^{r e f}
\end{array}\right|^{T}=\left|\begin{array}{l}
\dot{x}_{p}^{\text {ref }}(0), \cdots, \dot{x}_{p}^{\text {ref }}(\hat{s}), \cdots, \dot{x}_{p}^{\text {ref }}(1) \\
\dot{y}_{p}^{\text {ref }}(0), \cdots, \dot{y}_{p}^{\text {ref }}(\hat{s}), \cdots, \dot{y}_{p}^{r e f}(1)
\end{array}\right|^{T}
\end{aligned}
$$

Furthermore, our robot has control references for each actuator as detailed in (3), which realize the designed standing motion (2). $\dot{\mathbf{x}}_{r b t}^{r e f}$ is the motion reference for a powered walker and $\dot{\mathbf{y}}_{r b t}^{r e f}$ is for a standing assistance manipulator.

$$
\mathbf{v}_{r b t}^{r e f}=\left|\begin{array}{l}
\dot{\mathbf{x}}_{r b t}^{r e f} \\
\dot{\mathbf{y}}_{r b t}^{r e f}
\end{array}\right|^{T}=\left|\begin{array}{l}
\dot{x}_{x r t}^{r e f}(0), \cdots, \dot{x}_{r b t}^{r e f}(\hat{s}), \cdots, \dot{x}_{r b t}^{r e f}(1) \\
\dot{y}_{r b t}^{r e f}(0), \cdots, \dot{y}_{r b t}^{r e f}(\hat{s}), \cdots, \dot{y}_{r b t}^{r e f}(1)
\end{array}\right|^{T}
$$

- Second, we assumed the subject applies all forces $\mathbf{f}_{\text {user }}$ at position $\mathrm{P}$ because the armrest and the handle of our assistive robot are connected rigidly. We can calculate $\mathbf{f}_{\text {user }}$ from the force applied to the armrest $\mathbf{f}_{\text {armrest }}$ and the handle $\mathbf{f}_{\text {handle }}$ using force sensors in the robot's body (Fig.5(a)) as (4).

$$
\mathbf{f}_{\text {user }}=\mathbf{f}_{\text {armrest }}+\mathbf{f}_{\text {handle }}
$$

- Third, we assumed the patient also applies a force for doing a voluntary movement of their own intention, therefore $\mathbf{f}_{\text {user }}$ shows a voluntary component. At the same time, our controller calculates a motion reference $\mathbf{v}_{p}^{\text {ref }}$ at this posture (Fig.4(b)) and refers its investigated tolerance (gray area at Fig.4(b)). Our controller evaluates if $\mathbf{f}_{\text {user }}$ is within the tolerance at this posture, the patient's motion fulfills the both conditions as discussed in section two. 
- Finally, our robot controls two actuators by (5).

$$
\begin{aligned}
& v_{r b t}^{\text {upref }}=\left|\begin{array}{c}
\dot{x}_{r b t}^{\text {upref }} \\
\dot{y}_{r b t}^{u p r e f}
\end{array}\right|^{T} \\
& =\left|\begin{array}{c}
\dot{x}_{r b t}^{r e f}-\frac{B}{j}\left(f_{\text {handle }}-f_{\text {handle } 0}\right)-K\left(x_{r b t}-x_{r b t}^{r e f}\right) \\
\dot{y}_{r b t}^{r e f}-B\left(f_{\text {armrest }}-f_{\text {armrest } 0}\right)-K\left(y_{r b t}-y_{r b t}^{r e f}\right)
\end{array}\right|
\end{aligned}
$$

where $\mathbf{v}_{r b t}^{\text {upref }}$ is the updated reference value that our robot actually uses for delivering standing assistance. $\left(x_{r b t}, y_{r b t}\right)$ is the actual position of the powered walker and the standing assistance manipulator of our robot. $B$ and $K$ in (5) are constants used to coordinate the ratio between the damping and position controls. $j$ is also a constant which reduces the damping control ratio of the powered walker because it does not reduce the patient's load, and on the other hand, it largely affects the body balance. $f_{\text {handleo }}$ and $f_{\text {armrest } 0}$ are the forces the patient applies to the assistance system before he or she stands.

In order to apply the damping control only when the patient's motion fulfills both the stability condition and the muscle condition, the coefficient $B$ that validates the damping control mode is calculated as (6). $B$ will be larger value if $\mathbf{f}_{\text {user }}$ locates on the center of the tolerance and in this situation, it fits $\mathbf{v}_{p}^{r e f}$.

$$
\left\{\begin{array}{c}
\left.B=b \frac{\mathbf{v}_{p}^{r e f} \cdot \mathbf{f}_{\text {user }}}{\left|\mathbf{v}_{p}^{\text {ref }}\right| \cdot\left|\mathbf{f}_{\text {user }}\right|} \quad \text { (if conditions are fulfilled }\right) \\
B=0 \quad(\text { if conditions are not fulfilled })
\end{array}\right.
$$

By contrast, the position control is always useful because it helps the patient maintain a stable posture during motion. Therefore, we set the coefficient, $K$ which validates the position control mode, to be constant. The values of $b$ and $K$ were determined experimentally. Using these ideas, our controller sets the ratio of the damping control mode to a larger value if the patient's trajectory fits the expected reference pathway. Thus, the patient can move freely as intended if their posture is not largely different from the reference posture.

\section{Experiments}

We implemented our proposed idea to the prototype (Fig. 1(a)) and conducted a practical experiment with it. To confirm the efficiency of our pro- 


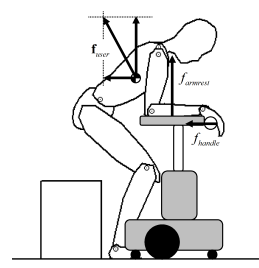

(a) Applied force and body movement vector

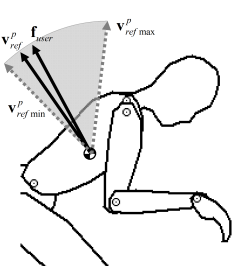

(b) Body motion and its range

Fig. 5. Voluntary movement during standing

posed assistance control scheme, we tested three cases.

- Case1: Using only position control, without our proposed idea.

- Case2: Using our proposed idea (Fig. 6(a)).

- Case3: The same as case 2, but in this case, the subject tries to move in a forward direction away from the designed standing motion reference pathway (Fig. 6(b)).

We used five subjects and each subject attempted all three cases, five times each. All subjects were young students who wore special equipment to mimic the experience of the elderly, ${ }^{13}$ limiting the testers body motions.

As seen in Fig. 6, our prototype succeeds to assist the standing motion even if the subject does not fit the reference pathway.

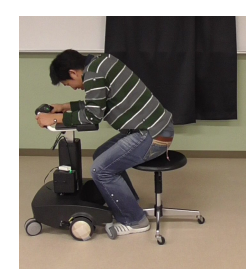

(a) Subject A, Case2

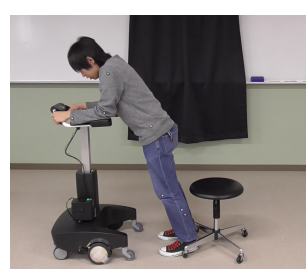

(b) Subject B, Case3

Fig. 6. Standing motion achieved using our proposed idea

Fig. 7(a) shows the variation in position of point $\mathrm{P}$ of subject $\mathrm{A}$ and Fig. 7(b) shows the electromyography (EMG) of the biceps femoris muscle that has been normalized by the maximum voluntary contraction. In case 1 , the subject's position fits the reference trajectory and this means our robot does not allow the patient to move freely as intended. As a result, the robot applies all required force for standing up to the subject and the subject is not require to use their own physical strength, as Fig. 7(b). 
On the other hand, in case 2 , the subject's position does not fit the reference trajectory but is within the investigated tolerance range. This mean our robot evaluates that the patient's motion fulfills both the stability and muscle conditions, and accepts the body motion of the patient even though it does not fit the reference pathway. As the result, the robot does not apply any unnecessary assistive force and succeeds in allowing the subject to use their remaining physical strength during the process of standing up.

In case 3 , once again the subject's position does not fit the reference trajectory and the subject tries to exceed the margin of tolerance. At points when the subject moves within the tolerance range, our robot assists the patient in the same manner as in case 2 . However, as seen by the dashed circle in Fig. 7(a), when the subject's position exceeds the tolerance trajectory and the patient's motion does not fulfill both the stability and muscle conditions, our robot changes the level of assistance control and as a result, the subject's position is returned to within the tolerance range. Furthermore, even in case 3, our robot allows the subject to use their own physical strength, in contrast to case 1.

According to these results, our robot succeeds to provide assistance to subjects while also allowing them to use their own physical strength. Moreover our robot maintains the body balance during the standing up process and our idea realizes a safe method of robotic assistance.

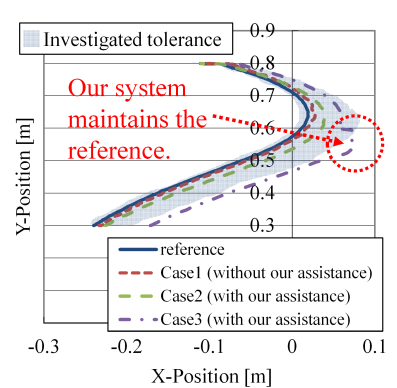

(a) P position (Subject $\mathrm{A}$ )

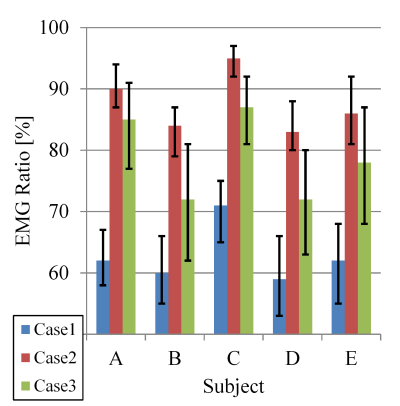

(b) Maximum EMG during standing

Fig. 7. Experimental results

\section{Conclusion}

This paper proposes a novel standing assistance device, which allows patients to maximize the use of their physical strength. To realize this, we investigated the motion tolerance of the standing posture, which fulfills 
both body balance and muscle force conditions. Furthermore, we proposed a novel assistance control, which maintains body stability whilst using physical strength. We conducted practical experiments to confirm the efficiency of the proposed idea implemented in our prototype of a robotic standing assistance device.

\section{Acknowledgments}

This work is supported in part by Grant-in-Aid for Scientific Research C (16K01580) from Japan Society for the Promotion of Science and the ASTEP functional verification phase (VP29117940231) from Japan Science and Technology Agency.

\section{References}

1. N. B. Alexander, A. B. Schultz and D. N. Warwick, J. of Geometry: MEDICAL SCIENCES 46, M91 (1991).

2. M. A. Hughes and M. L. Schenkman, J. of Rehabilitation Research and Development 133, 409 (1996).

3. C. Office, KOUREISHA HAKUSHO (The whitepaper on the aged society, written in Japanese) (Government of Japan, 2011).

4. M. Hirvensalo, T. Rantanen and E. Heikkinen, J. of the American Geriatric Society 48, 493 (2000).

5. K. Nagai, I. Nakanishi and H. Hanabusa, Assistance of self-transfer of patients using a power-assisting device, in Proc. IEEE/RAS-EMBS Int. Conf. on Robotics and Automation (ICRA'03), (Taipei, Taiwan, 2003).

6. A. Funakubo, H. Tanishiro and Y. Fukui, J. of the Society of Instrument and Control Engineers 40, 391 (2001).

7. T. Yamada, M. Sakaguchi, D. Chugo, S. Yokota and H. Hashimoto, A load estimation of a patient considering with a posture during standing motion, in Proc. Int. Conf. on Climbing and Walking Robots (CLAWAR'14), (Poznan, Poland, 2014).

8. D. Chugo, S. Muramatsu, S. Yokota and H. Hashimoto, A standing assistance for both voluntary movement and postural adjustment, in Proc. Int. Conf. on Climbing and Walking Robots (CLAWAR'16), (London, UK, 2016).

9. S. Kawazoe, D. Chugo, S. Muramatsu, S. Yokota, H. Hashimoto, T. Katayama, Y. Mizuta and A. Koujina, Pattern based standing assistance for a low level of care, in Proc. Int. Conf. on Climbing and Walking Robots (CLAWAR'17), (Porto, Portugal, 2017).

10. Opensim documentation gait 2392 and 2354 models (2018), https://simtk-confluence.stanford. edu/display/OpenSim/Gait+2392+and+2354+Models.

11. H. Okada, M. Ae, N. Fujii and Y. Morioka, Biomechanisms 13, 125 (1996).

12. T. Ito, T. Azuma and N. Yamashita, Europian J. of Applied Physiology 92, 186 (2004).

13. K. Takeya, Y. Kanemitsu and Y. Futoyu, Kawasaki Medical Welfare J. 11, $64(2001)$. 